\title{
SOBRE DIFICULDADES DE INGRESSANTES NO ENSINO MÉDIO NA COMPREENSÃO DE NÚMEROS RACIONAIS ${ }^{1}$
}

\author{
Alexia Bezerra de Carvalho ${ }^{2}$ \\ Rita de Cássia da Costa Guimarães \\ William Vieira \\ Emanoel Fabiano Menezes Pereira \\ Roberto Seidi Imafuku
}

\begin{abstract}
RESUMO
Apresenta-se, neste artigo, uma análise da resolução de uma questão sobre números racionais, exposta por estudantes ingressantes no Ensino Médio. O objetivo foi identificar as principais dificuldades e erros apresentados pelos participantes sobre o conceito e operações de números racionais. Para isso, realizou-se uma análise de erros das resoluções apresentadas pelos participantes. A interação de aspectos algorítmicos, intuitivos e formais é o referencial teórico adotado nas análises. Verificou-se que os estudantes não possuem clareza sobre o conceito de número racional e apresentam, em decorrência, dificuldades no uso dos procedimentos algorítmicos necessários para a resolução da questão proposta.
\end{abstract}

Palavras-chave: Números racionais. Análise de erros. Dificuldades. Interpretação.

\section{DIFFICULTIES OF HIGH SCHOOL ENTRANTS IN UNDERSTANDING RATIONAL NUMBERS}

\begin{abstract}
This paper presents an analysis of the resolution of a question about rational numbers, posed by students entering high school. The goal was to identify the main difficulties and errors presented by the participants about the concept and operations of rational numbers. To do so, an error analysis of the resolutions presented by the participants was performed. The interaction of algorithmic, intuitive and formal aspects is the theoretical framework adopted in the analysis. It was found that students do not have clarity about the concept of rational numbers and present, as a result, difficulties in the use of algorithmic procedures necessary to solve the proposed question.
\end{abstract}

Keywords: Rational numbers. Error analysis. Difficulties. Interpretation.

\footnotetext{
${ }^{1}$ Como citar este artigo: CARVALHO, Alexia Bezerra; GUIMARÃES, Rita de Cássia da Costa; VIEIRA, William; PEREIRA, Emanoel Fabiano Menezes; IMAFUKU, Roberto Seidi. Sobre dificuldades de ingressantes no ensino médio na compreensão de números racionais. ForScience, Formiga, v. 9, n. 1, e00878, jan./jun. 2021. DOI: 10.29069/forscience.2021v9n1.e878.
}

${ }^{2}$ Autor para correspondência: Alexia Bezerra de Carvalho, e-mail: alexiabc16@gmail.com 


\section{INTRODUÇÃO}

As preocupações com o ensino e aprendizagem dos números racionais estão presentes nos documentos oficiais que norteiam o ensino de Matemática no Brasil. Os Parâmetros Curriculares Nacionais ( $\mathrm{PCN}$ ) destacam que a dificuldade com o trabalho com números racionais persiste até no Ensino Superior, no qual os estudantes chegam sem compreender o significado desses conceitos e com dificuldades com os cálculos (BRASIL, 1997), e indicam que essas dificuldades podem prejudicar o avanço dos estudantes nos estudos. Mais recentemente, a Base Nacional Comum Curricular (BRASIL, 2017) destaca que para os anos finais do Ensino Fundamental é esperado que os “(...) estudantes consigam resolver problemas com números racionais envolvendo operações fundamentais, com seus diferentes significados, e utilizando estratégias diversas, com compreensão dos processos neles envolvidos" (BRASIL, 2017, p. 225).

Esses documentos oficiais destacam que, além do conteúdo ser importante para o avanço do desenvolvimento de outros temas do currículo de Matemática, o trabalho com números racionais deve abordar as diferentes representações e não se ater somente aos algoritmos de operações que, por si só, não tem significado para os estudantes (BRASIL, 1997, 2017).

A relevância deste tema para a formação dos estudantes da Educação Básica tem levado pesquisadores brasileiros e internacionais a investigarem as dificuldades relacionadas aos processos de ensino e de aprendizagem de números racionais ao longo dos anos. Por exemplo, Guerreiro e Serrazina (2017), em estudo desenvolvido com 42 estudantes de 8 a 10 anos de uma escola pública da grande Lisboa, discutem sobre o desenvolvimento do sentido de números racionais na aprendizagem deste tema. Segundo as autoras, no que diz a respeito à aprendizagem dos números racionais "(...) os alunos revelam dificuldade na compreensão da rede de conceitos que o estudo destes números envolve" (GUERREIRO; SERRAZINA, 2017, p. 182). As pesquisadoras ressaltam a importância do trabalho com diferentes representações dos números racionais, destacando que a visualização pode ser um facilitador da construção intuitiva do significado de razão.

Monteiro e Groenwald (2014) investigaram as dificuldades na aprendizagem de números racionais de 19 estudantes do $7^{\circ}$ ano do Ensino Fundamental em testes adaptativos envolvendo o conteúdo de frações. Para isso, foi desenvolvida uma sequência didática com a utilização de recursos tecnológicos com atividades online disponibilizados para os estudantes no Sistema Integrado de Ensino e Aprendizagem (SIENA). Os resultados apontaram que os 
participantes apresentaram dificuldades nos testes envolvendo o conceito, equivalência, simplificação e comparação de frações, mas tiveram um bom desempenho nos testes envolvendo adição, subtração, multiplicação e divisão com frações.

Santos e Fonseca (2019) investigaram as dificuldades dos estudantes do $7^{\circ}$ ano do Ensino Fundamental de uma escola municipal da zona rural de São Sebastião/AL na aprendizagem de fração. Foram aplicadas três questões abertas com problemas contextualizados, envolvendo operações de adição e subtração de frações e uma questão para calcular o resultado das quatro operações fundamentais com frações. Os resultados indicaram que os estudantes possuíam dificuldades no entendimento do conceito de fração, relação parte-todo, operações fundamentais com frações e no cálculo do MMC para adição e subtração com denominadores diferentes. As pesquisadoras destacam que “(...) se faz necessário ter um acompanhamento da compreensão e aprendizagem dos estudantes no conteúdo de fração, visando sempre uma forma de contribuir significativamente para a superação das dificuldades encontradas" (SANTOS; FONSECA, 2019, p. 65).

Freire e Lima (2019) elaboraram e aplicaram um questionário para 41 estudantes do $6^{\circ}$ ano do Ensino Fundamental com o objetivo de analisar características formais presentes no trabalho destes participantes com os números racionais, nos subconstrutos denominados parte-todo e medida. A aplicação das questões aconteceu em dois momentos, antes e depois dos estudantes aprenderem números racionais na forma fracionária com o professor titular de Matemática. Os resultados apontaram uma melhora de alguns estudantes entre a primeira e a segunda coleta, entretanto a maioria dos participantes indicou ter dificuldades relacionadas à falta de entendimento de características formais em relação aos dois subconstrutos explorados.

Neste artigo, apresentamos a análise das resoluções de uma questão relacionada ao conteúdo de números racionais presente em um questionário diagnóstico. O questionário foi aplicado para 70 estudantes ingressantes no Ensino Médio Técnico, teve duração de uma hora e meia e foi realizado de maneira individual na Instituição de ensino na qual os participantes estudam. O questionário possui doze questões que abordam temas do Ensino Fundamental. A análise de erros proposta por Cury (2007) é o procedimento utilizado na classificação das dificuldades identificadas nas resoluções dos participantes e a interação de aspectos intuitivos, algorítmicos e formais colocados por Fischbein (1994) é o referencial teórico adotado nas análises.

\section{FUNDAMENTAÇÃO TEÓRICA}


Apoiamo-nos nas ideias de Fischbein (1994) sobre a interação de aspectos intuitivos, algorítmicos e formais quando os estudantes buscaram estratégias para resolver problemas em Matemática. Este pesquisador considera a interação destes aspectos fundamental para a consideração da Matemática como atividade humana e para entendê-la não apenas como um conjunto de procedimentos e técnicas, mas como um processo criativo.

Segundo Fischbein (1994), aspectos intuitivos estão associados a uma compreensão na qual o indivíduo considera um conhecimento como auto evidente, ou seja, que ele é capaz de compreender sem questionar a necessidade de argumentos convincentes para justificar a sua validade.

Os aspectos formais dizem respeito aos conhecimentos sobre as definições, axiomas, teoremas e provas que devem ser aprendidos, organizados e aplicados pelos estudantes. São eles que compõem o núcleo da Matemática como uma ciência formal. Para Fischbein (1994) é importante que se ofereça aos estudantes a possibilidade de acesso a um processo de aprendizagem que valorize a apropriação desse aspecto formal, posto que compreender o que seja rigor e coerência em Matemática não é uma tarefa que um estudante adquira de maneira espontânea, sem a mediação do professor.

Em relação aos aspectos algorítmicos, estes concernem às habilidades relacionadas às técnicas e procedimentos padronizados de resolução. Segundo Fischbein (1994, p. 232) “(...) é uma mera ilusão acreditar que ao saber axiomas, teoremas, provas e definições com exposto em livros-texto, a pessoa seria capaz de resolver problemas matemáticos". Desse modo, os aspectos algorítmicos constituem parte essencial do entendimento e da criação da Matemática. Em contrapartida, o domínio de técnicas e procedimentos, sem o conhecimento de justificativas, pode não ser suficiente para a resolução de problemas que fogem ao padrão.

Fischbein (1994) defende que deve existir uma interação entre os aspectos intuitivos, formais e algorítmicos na realização de qualquer atividade matemática.

As principais ideias que utilizamos nas análises dos dados obtidos são colocadas por Fischbein (1994), mas destacamos que a ideia de contrato didático pode ser identificada nas respostas apresentadas por alguns participantes, por isso definimos este conceito a seguir e o utilizamos nas análises.

O contrato didático refere-se ao conjunto de atitudes do professor que são esperadas pelos estudantes e o conjunto de atitudes que os estudantes esperam do professor. Ele “(...) é o conjunto de regras que determinam uma pequena parte explicitamente, mas sobretudo implicitamente, do que cada parceiro da relação didática deverá gerir e daquilo que, de uma maneira ou de outra, ele terá de prestar conta perante o outro" (BROUSSEAU, 1986 apud 
SILVA, 2008, p. 50).

\section{MATERIAL E MÉTODOS}

No início do ano letivo de 2019, foi elaborado e aplicado um questionário com doze questões para 70 estudantes ingressantes no Ensino Médio de uma Instituição de ensino pública federal, da região metropolitana de São Paulo. O objetivo foi explorar possíveis dificuldades em relação aos conteúdos de Matemática do Ensino Fundamental. As questões versavam sobre os temas Teorema de Pitágoras, Teorema de Tales, proporcionalidade, áreas, resolução de equações e de sistemas de equações, interpretação de Figuras e Tabelas, além de um problema envolvendo números racionais, sobre adição e subtração de frações.

A aplicação do questionário ocorreu no horário regular das aulas e foi feita pelo professor regente da turma. As questões foram resolvidas individualmente, em um período de uma hora e meia, no campus em que os participantes estudam. Nenhum tipo de consulta a materiais externos foi permitido e os pesquisadores não forneceram nenhuma informação adicional, além daquelas constantes nos questionários.

Os responsáveis pelos estudantes assinaram o Termo de Consentimento Livre e Esclarecido (TCLE). Os participantes assinaram o Termo de Assentimento de participação na pesquisa e são tratados por pseudônimos na discussão dos dados.

Como a questão que envolve números racionais foi a que apresentou menor índice de acertos. Neste trabalho, analisamos as resoluções para essa questão com o objetivo de entender quais os principais erros e dificuldades dos estudantes no que concerne às operações de adição e subtração de frações. As resoluções dos estudantes foram classificadas de acordo com a Análise de Erros proposta por Cury (2007), procedimento metodológico que é baseado em uma análise de conteúdo, na qual classes de erros recorrentes nas resoluções das questões são classificadas. Posteriormente, uma tabulação é feita, apresentando a frequência e o percentual de cada tipo de erro identificado.

\section{RESULTADOS E DISCUSSÃO}

A seguir, apresentamos os objetivos da questão proposta aos participantes, retirada da Prova Brasil 2011 - Matemática $8^{\mathrm{a}}$ série $/ 9^{\circ}$ ano e, a análise de suas resoluções.

Questão: A estrada que liga Recife a Caruaru será recuperada em três etapas. Na primeira 
etapa, será recuperado $\frac{1}{6}$ da estrada e na segunda etapa $\frac{1}{4}$ da estrada. Pergunta-se: que fração corresponde à terceira etapa?

Com esta situação-problema, procuramos explorar o conhecimento dos estudantes sobre adição e subtração dos números racionais expressos na forma fracionária. O objetivo com a questão foi investigar se os participantes inter-relacionam aspectos algorítmicointuitivos (FISCHBEIN, 1994) associados às operações com frações com aspectos formais relacionados à identificação do todo como uma unidade que pode ser expressa na forma de uma fração.

A análise das resoluções mostrou que 7,1\% dos 70 participantes acertaram a questão, índice que revela dificuldades da maioria dos ingressantes na interpretação de problemas desse tipo. A questão pode ser resolvida fazendo uso da adição e subtração de frações, conforme exemplificado na resolução de Renato (Figura 1).

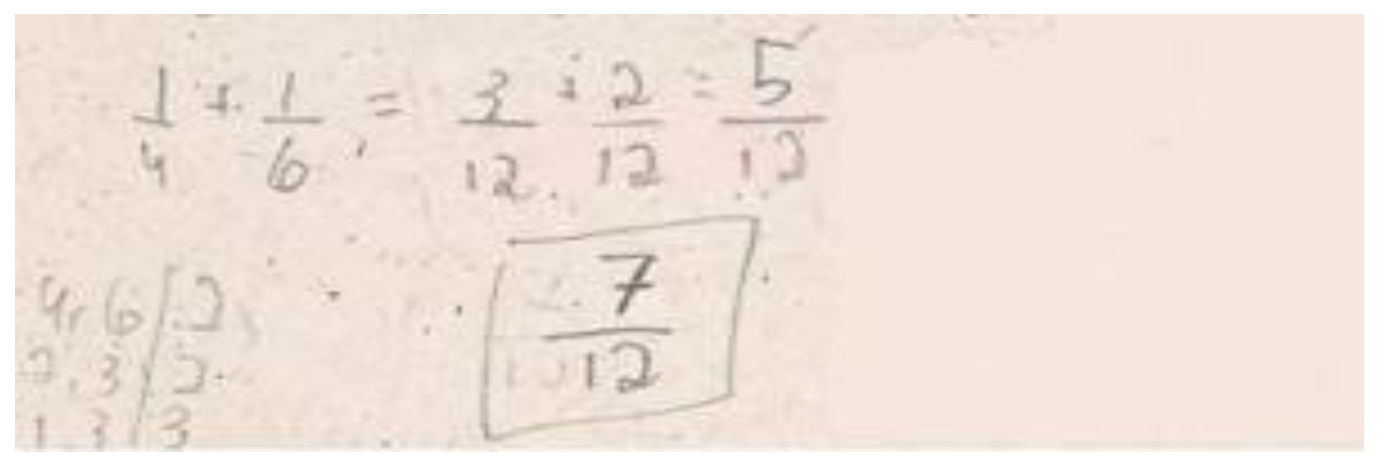

Figura 1 - Resposta de Renato

Fonte: Autores (2020).

Embora não tenha explicitado seus cálculos, é possível inferir que Renato compreende que toda a estrada, neste caso, pode ser identificada como uma fração do tipo $\frac{12}{12}$, o que levou este participante a obter os $\frac{7}{12}$ referentes à terceira etapa da recuperação da estrada. Também cumpre observar que Renato não apresenta um texto escrito para indicar sua resposta e limitase a circular a fração encontrada como solução do problema. Dessa forma, entendemos que, apesar deste estudante apresentar bons conhecimentos sobre o tema e na interpretação do problema, ainda precisa avançar na comunicação de suas ideias, tarefa que, acreditamos, deveriam ter sido assimiladas ao longo do Ensino Fundamental.

A quantidade de estudantes que erraram a questão foi de 65. Para as respostas consideradas erradas, apresentamos as classes de erros identificadas no Quadro 1. 


\begin{tabular}{|l|c|c|}
\hline \multicolumn{1}{|c|}{ Descrição do erro } & Freq & $\%$ \\
\hline $\mathrm{A}_{1}-$ Realizar subtração de $\frac{1}{6} \mathrm{e} \frac{1}{4}$, encontrando $\frac{1}{2}$ & 8 & $12,3 \%$ \\
\hline $\mathrm{B}_{1}-$ Estratégias equivocadas & 9 & $13,8 \%$ \\
\hline $\mathrm{C}_{1}-$ Sem resolução & 43 & $66,2 \%$ \\
\hline $\mathrm{D}_{1}-$ Em branco & 5 & $7,7 \%$ \\
\hline
\end{tabular}

Quadro 1 - Erros identificados na resolução da questão proposta

Fonte: Autores (2020).

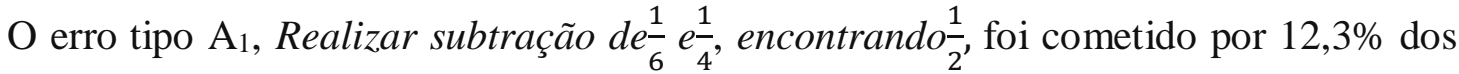
participantes. Monteiro e Groenwald (2014), também identificam que os participantes de sua pesquisa realizaram "a soma independentemente dos numeradores e denominadores" (MONTEIRO; GROENWALD, 2014, p. 125).

As respostas de João e Marcelo, destacadas na Figura 2, são exemplos desse tipo de erro, pois esses participantes realizaram a subtração de $\frac{1}{6} \mathrm{e} \frac{1}{4}$, encontrando $\frac{1}{2}$, evidenciando uma confusão com aspectos intuitivos (FISCHBEIN, 1994) relacionados à interpretação das informações dadas no enunciado da questão.

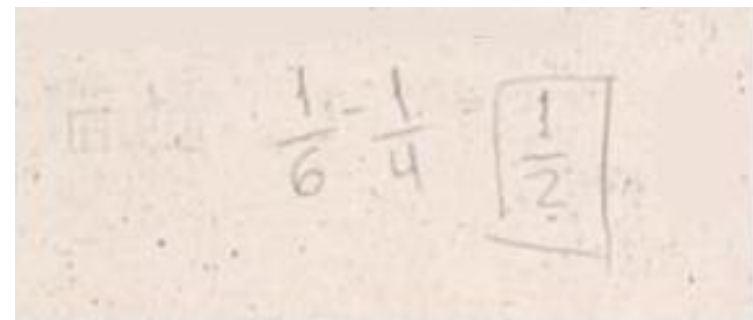

(a)

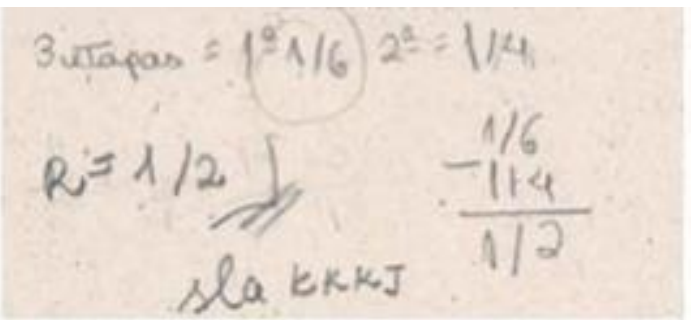

(b)

Figura 2 - Respostas de João e Marcelo para a questão proposta Fonte: Autores (2020)

Conforme destacado na imagem na Figura 2 (a), João realizou a subtração das frações sem utilizar um algoritmo correto, indicando que o estudante não possui o conhecimento necessário para efetuar a resolução. Em sua resolução, o estudante Marcelo (Figura 2 (b)) utiliza o algoritmo da subtração de números racionais na forma decimal para efetuar a operação e, com isso, demonstra que ainda não possui clareza sobre aspectos formais relacionados ao conceito de fração e sobre aspectos algorítmicos (FISCHBEIN, 1994) relacionados às operações de subtração de frações. Ao utilizar a subtração a fim de encontrar 
a resposta é perceptível a não compreensão de conceitos relacionados ao significado de fração como parte-todo. No que concerne às dificuldades relacionadas ao conteúdo de parte-todo, Freire e Lima (2019) indicam que há a "necessidade de se discutir características formais no trabalho com esse subconstruto" (FREIRE; LIMA, 2019, p. 50).

O fato do referido participante tentar aplicar um algoritmo usado para a subtração de números decimais para a subtração de frações indica que a extensão de aspectos algorítmicointuitivos que não estão inter-relacionados a aspectos formais (FISCHBEIN, 1994) associados à subtração de números decimais podem levar os estudantes a interpretações equivocadas e a cometerem erros como este. Dessa maneira, entendemos que, o algoritmo se torna algo estéril e estabelece aspectos intuitivos (FISCHBEIN, 1994) equivocados, que impingem aos estudantes ideias confusas sobre conceitos e o uso das operações.

No caso da opção pela operação de subtração, aparentemente os participantes se valem de aspectos intuitivos (FISCHBEIN, 1994) relacionados à "terceira etapa" destacada no enunciado do problema, e a relacionaram à parte que falta; contudo, ao não interrelacionarem essas ideias a aspectos formais (FISCHBEIN, 1994) associados à ideia de todo (toda a estrada), não foram capazes de apresentar uma resolução para o problema.

O erro tipo B1, Estratégias equivocadas, foi cometido por 13,8\% dos participantes e representa uma variedade de tentativas de responder à questão utilizando cálculos quaisquer. Dentre os erros deste tipo, as respostas de Eduardo e Mônica, destacadas na Figura 3, foram as que mais chamaram atenção por apresentarem mais possibilidades de discussão.

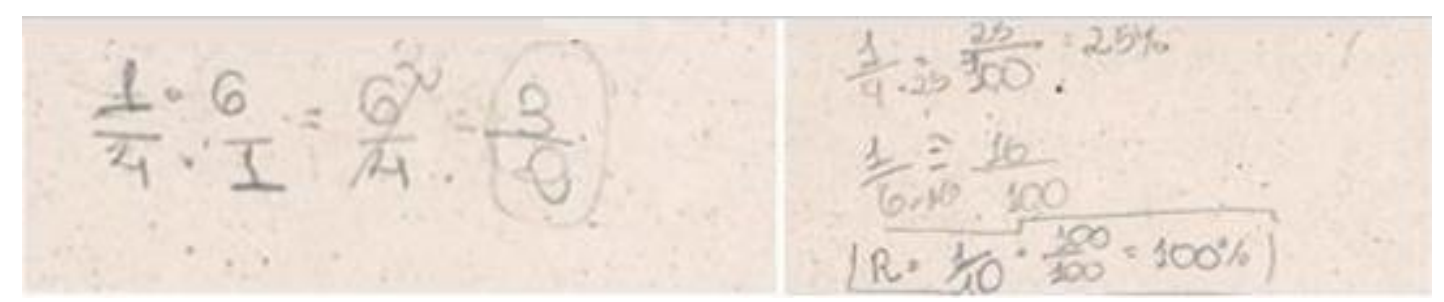

(a)

(b)

Figura 3 - Respostas de Eduardo e Mônica para a questão proposta Fonte: Autores (2020).

Em sua resposta, na Figura 3(a), Eduardo utiliza a operação de divisão de frações para obter o resultado, indicando uma estratégia equivocada e que não nos permite identificar as razões que levaram o estudante a realizá-la. Também podemos destacar o que parece ser a influência do contrato didático (SILVA, 2008), na qual o estudante, não querendo deixar a questão em branco, tenta resolvê-la de alguma maneira aleatória. A estudante Mônica tenta utilizar a relação das frações com a porcentagem para resolver a questão proposta (ver Figura 
3(b)). A participante encontra a representação na forma de porcentagem para $\frac{1}{4}$ fazendo uso de frações equivalentes, porém não obtém êxito ao tentar realizar o mesmo procedimento para a fração $\frac{1}{6}$. A resposta final apresentada pela participante parece não ter relação com os cálculos realizados e também não nos permite identificar seus motivos. Além disso, a estudante comete um erro ao igualar as frações $\frac{1}{10}$ e $\frac{100}{100}$. Nesse caso, apontamos para a não compreensão de aspectos formais (FISCHBEIN, 1994), que estão relacionados ao conceito de equivalência de frações. As dificuldades em entender conceitos relacionados ao estudo de números racionais também são apontadas por Guerreiro e Serrazina (2017).

O erro tipo C1, Sem resolução, foi o mais recorrente nas respostas, tendo sido cometido por $66,2 \%$ dos estudantes. Nessa categoria, foram incluídas respostas que apresentaram apenas um número, sem exibir os cálculos, perspectiva que indica um possível "chute" por parte dos participantes. A resposta de Jeremias, na Figura 4 (b), é um exemplo dessa situação. Dos 43 estudantes que cometeram esse erro, 36 chegaram ao resultado $\frac{1}{2}$, e levantamos a hipótese de que esses participantes podem ter realizado uma subtração entre os denominadores 6 e 4 das frações $\frac{1}{6}$ e $\frac{1}{4}$, mas não conseguimos ter certeza se foi isso que aconteceu, pois os estudantes não exibiram seus cálculos. A resposta de Pablo, destacada na Figura 4 (a), exemplifica essa situação.

(a)

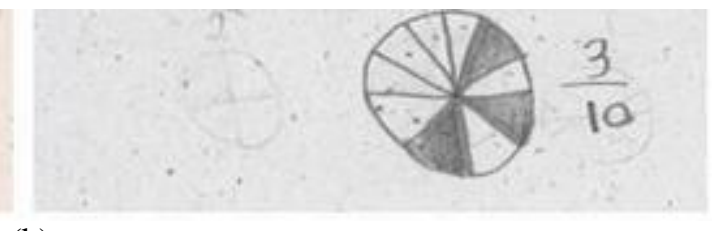

(b)

Figura 4 - Respostas de Pablo e Jeremias para a questão proposta Fonte: Autores (2020).

Destacamos que o fato de os estudantes terem colocado uma resposta, mesmo sem apresentar a resolução, revela um imperativo que os faz não deixar a questão em branco. Em sua investigação, Santos e Fonseca (2019) também identificam erros que “(...) se devem ao fato de que, simplesmente, o aluno não sabe a resposta e escreve uma resposta qualquer só para não deixar em branco a questão" (SANTOS; FONSECA, 2019, p. 65). Essa situação pode indicar que, de maneira implícita, há a ação do contrato didático (SILVA, 2008) em relação ao comportamento dos estudantes que seria esperado pelos professores, fazendo com que os participantes não deixem a questão sem resposta.

Os $7,7 \%$ dos participantes que deixaram a questão em branco, erro tipo $\mathrm{D}_{1}$, ajudam a 
corroborar o quadro de muitas dificuldades dos ingressantes.

\section{CONSIDERAÇÕES FINAIS}

Neste artigo, tivemos como objetivo identificar as principais dificuldades e erros sobre o conceito e as operações com frações presentes nas resoluções de ingressantes no Ensino Médio Técnico, de forma a compreender se, e como, estes inter-relacionam aspectos algorítmicos, intuitivos e formais (FISCHBEIN, 1994) em suas estratégias de resolução. A questão proposta envolvia adição e subtração de números racionais na forma de fração e o trabalho com a relação parte-todo. As análises revelam que os estudantes obtiveram um desempenho abaixo do esperado para ingressantes no Ensino Médio, posto que, segundo documentos oficiais brasileiros para o ensino de Matemática (BRASIL, 1997; 2017), conteúdos relacionados aos números racionais, como operações e mudança entre representações, deveriam ser dominados ao final do Ensino Fundamental.

Chama atenção em nossa investigação o alto índice de respostas que não apresentaram justificativas ou cálculos e as questões em branco, que correspondem a 66,2\% do total de respostas. Por outro lado, os participantes que apresentaram tentativas de resolução da questão revelaram muitas dificuldades com aspectos formais relacionados ao conceito de fração e também com aspectos algorítmico-intuitivos associados ao uso de algoritmos de subtração de frações e à interpretação das informações presentes no enunciado da questão proposta. $\mathrm{O}$ uso equivocado de algoritmos de subtração de frações e a extensão de técnicas de subtração de números decimais para frações revelam a não interação de aspectos algorítmicos e formais relacionados ao aprendizado dessas técnicas.

Nesse sentido, nossos resultados estão em consonância tanto com os colocados por Monteiro e Groenwald (2014), que apontam dificuldades dos estudantes com aspectos conceituais no estudo de números racionais, quanto com as posições de Guerreiro e Serrazina (2017) que destacaram que houve dificuldades na compreensão da rede de conceitos que abrange o estudo dos números racionais, por parte de seus sujeitos de pesquisa.

Assim como em trabalhos de Santos e Fonseca (2019), nos quais são destacados aspectos relativos a dificuldades dos estudantes em trabalhar com frações, nosso estudo também indicou que há dificuldades em relação à utilização de algoritmos das operações fundamentais com frações. Além disso, foi possível perceber a falta de compreensão das características formais na relação parte-todo. Esses resultados corroboram a perspectiva destacada por Freire e Lima (2019) em relação às dificuldades no entendimento dos conceitos 
formais desse tipo de abordagem do conceito de fração.

De maneira geral, sustentamos que para o sucesso do aprendizado é necessário um ensino que proporcione a interação de aspectos formais, algorítmicos e intuitivos presentes nas ideias e conceitos matemáticos. Esperamos que os resultados obtidos possam colaborar para um repensar sobre os processos de ensino e aprendizagem de números racionais e da Matemática na Educação Básica.

\section{REFERÊNCIAS}

BRASIL. Parâmetros curriculares nacionais: matemática. Brasília: MEC, 1997.

BRASIL. Base nacional comum curricular. 3. ed. Brasília: MEC, 2017.

CURY, H. N. Análise de erros: o que podemos aprender com as respostas dos alunos. Belo Horizonte: Autêntica, 2007.

FISCHBEIN, E. The interaction between the formal, the algorithmic and the intuitive components in a mathematical activity. In:BIEHLER, R. et al. (Org.) Didactics of mathematics as a scientific discipline. Dordrecht: Kluwer Academic Publisher, 1994. p. 328-375.

FREIRE, P. C.; LIMA, R. N. Números racionais na forma fracionária: a influência de características formais. Revista Sergipana de Matemática e Educação Matemática. Itabaiana, v. 1, p. 37-51, 2019. Disponível em: https://pontadelanca.revistas.ufs.br/index.php/ReviSe/article/view/10271. Acesso em: 25 jan. 2020

GUERREIRO, H. G.; SERRAZINA, M. L. Aprendizagem dos números racionais com compreensão envolvendo um processo de modelação emergente. Bolema, Rio Claro, v. 31, n. 57, p. 181-201, abr. 2017. Disponível em: https://www.scielo.br/scielo.php?pid=S0103636X2017000100011\&script=sci_arttext. Acesso em: 18 jan. 2020.

MONTEIRO, A. B.; GROENWALD, C. L. O. Dificuldades na aprendizagem de frações: reflexões a partir de uma experiência utilizando testes adaptativos.

Alexandria: Revista de Educação em Ciência e Tecnologia, Florianópolis, v. 7, n. 2, p. 103-135, 2014. Disponível em:

https://periodicos.ufsc.br/index.php/alexandria/article/view/38217. Acesso em: 24 out. 2019.

SANTOS, R.; FONSECA, S. S. Dificuldades dos alunos do $7^{\circ}$ ano do ensino fundamental em aprender fração. Revista Insignare Scientia, Cerro Largo, v. 2, n. 1, p. 50-66, jan./abr. 2019. Disponível em: https://periodicos.uffs.edu.br/index.php/RIS/article/view/10724. Acesso em: 14 nov. 2019.

SILVA, B. A. Contrato didático. In: MACHADO, S. D. A. (Org.), Educação matemática: uma (nova) introdução. São Paulo: EDUC, 2008. p. 49-75. 


\title{
DADOS DOS AUTORES
}

\author{
Alexia Bezerra de Carvalho \\ E-mail: alexiabc16@gmail.com \\ Currículo Lattes: http://lattes.cnpq.br/3931770740526666 \\ Licencianda em Matemática pelo Instituto Federal de São Paulo (IFSP) Campus Guarulhos.
}

\section{Rita de Cássia da Costa Guimarães}

E-mail: rdrita.cg@gmail.com

Currículo Lattes: http://lattes.cnpq.br/6415268784802481

Licencianda em Matemática pelo Instituto Federal de São Paulo (IFSP) Campus Guarulhos. Membro do Grupo de Estudos e Pesquisa em Educação Matemática e Formação de Professores (GEPEMFOP do IFSP) - Campus Guarulhos.

\section{William Vieira}

E-mail: wvieira@ifsp.edu.br

Currículo Lattes: http://lattes.cnpq.br/6106510148543215

Doutor em Educação Matemática pela Universidade Anhanguera de São Paulo, mestrado e licenciatura em Matemática pelo Instituto de Matemática e Estatística da Universidade de São Paulo. Professor do Instituto Federal de São Paulo (IFSP) Campus Guarulhos. Membro do Grupo de Estudos e Pesquisa em Educação Matemática e Formação de Professores (GEPEMFOP do IFSP) - Campus Guarulhos.

\section{Emanoel Fabiano Menezes Pereira}

E-mail: emanoel.pereira@ifsp.edu.br

Currículo Lattes: http://lattes.cnpq.br/4601893345601275

Mestre em Matemática pela Universidade Federal do ABC (UFABC) Campus Santo André, especialista em Educação à Distância pela Universidade Federal Fluminense (UFF) e graduado em Licenciatura em Matemática pela Universidade de São Paulo (USP). Atualmente é professor efetivo do Instituto Federal de Educação, Ciência e Tecnologia de São Paulo (IFSP), é orientador e preceptor do Programa de Residência Pedagógica - área de Matemática do IFSP/Campus Guarulhos.

\section{Roberto Seidi Imafuku}

E-mail: roberto.imafuku@ifsp.edu.br

Currículo Lattes: http://lattes.cnpq.br/7926295090638853

Doutor em Educação Matemática pela Universidade Anhanguera de São Paulo, mestrado acadêmico em Educação Matemática pela Pontifícia Católica (PUC). Professor do Instituto Federal de São Paulo (IFSP) Campus Guarulhos. Membro do Grupo de Estudos e Pesquisa em Educação Matemática e Formação de Professores (GEPEMFOP do IFSP) - Campus Guarulhos. 